\title{
Concurrent task effects on memory retrieval
}

\author{
DOUG ROHRER \\ University of South Florida, Tampa, Florida \\ and \\ HAROLD E. PASHLER \\ University of California, San Diego, La Jolla, California
}

\begin{abstract}
Previous studies combining continuous free recall with a concurrent task have generally shown that concurrent tasks impose fairly negligible effects on memory retrieval. By contrast, dual-task studies employing either cued recall or semantic retrieval reveal gross memory impairment and suggest that retrieval is delayed by the centrally demanding phase of the concurrent tasks (i.e., response selection). To explore this conflict, subjects performed continuous free recall while carrying out a serial-choiceresponse time (RT) task, as in the previous free recall studies. Unlike these previous studies, however, the choice-RT task utilized arbitrary stimulus-response mappings in order to increase the proportion of time devoted to the centrally demanding response selection phase. Recall total was reduced significantly, and recall latency was slowed substantially.
\end{abstract}

People sometimes remark that it is subjectively taxing to retrieve information from memory, especially when such retrieval is not immediately successful. For example, it is tiring to search in vain for a forgotten name, and likewise, laboratory subjects complain of fatigue when asked to devote several minutes to the recall of a previously studied list of words. Intuitively, therefore, one might suppose that memory retrieval must impose demands on limited cognitive resources or mechanisms. However, this possibility is contradicted by a large number of recent laboratory studies revealing that free recall is scarcely affected when subjects must perform a demanding concurrent task while recalling a list of study words (Anderson, Craik, \& NavehBenjamin, 1998; Baddeley, Lewis, Eldridge, \& Thomson, 1984; Craik, Govoni, Naveh-Benjamin, \& Anderson, 1996; Craik, Naveh-Benjamin, Ishaik, \& Anderson, 2000; Iidaka, Anderson, Kapur, Cabeza, \& Craik, 2000; NavehBenjamin, Craik, Gavrilescu, \& Anderson, 2000; NavehBenjamin, Craik, Guez, \& Dori, 1998; Naveh-Benjamin, Craik, Perretta, \& Tonev, 2000; Naveh-Benjamin \& Guez, 2000). Although there are some exceptions to these results, they appear largely to be confined to studies requiring source memory (Troyer, Winocur, Craik, \& Moscovitch, 1999) or studies in which the concurrent task and the memory retrieval task both involve verbal materials (Fernandes \& Moscovitch, 2000; Park, Smith, Dudley, \&

This research was supported in part by National Institute of Mental Health Grant MH45584 to H.E.P. We thank Noriko Coburn for her invaluable assistance with the collection and analysis of the data, Joel Shotton and Trung Nguyen for their programming assistance, and Fergus Craik, Gordon Logan, and Jim Hoffman for their suggestions. Correspondence concerning this article should be addressed to D. Rohrer, Department of Psychology, PCD 4118G, University of South Florida, Tampa, FL 33620-7200(e-mail: drohrer@ chuma1.cas.usf.edu).
Lafronza, 1989). These exceptions notwithstanding, then, the studies cited above showed that free recall is mostly unaffected by a concurrent mental task.

The picture is complicated, however, by two studies in which memory paradigms other than free recall were used, because the results of these studies imply that memory retrieval is severely impaired by a concurrent task. In Carrier and Pashler (1995), single trials of cued recall were paired with a choice-response-time (RT) task. The results suggested severe competition between the retrieval and the concurrent task, with the memory retrieval apparently waiting for completion of processing in the concurrent task. Similarly, Rohrer, Wixted, Salmon, and Butters (1995) examined the impact of a concurrent task on the retrieval of category exemplars from semantic memory (e.g., kinds of fruits), and both the total number and the latency of these generated exemplars were grossly impaired by a serial-choice-RT task. The present article explores the apparent conflict between these two studies and the free recall studies by pairing free recall with a concurrent task that differs from those used in the previous free recall studies. In addition, the present study is the first to examine the effect of a concurrent task on recall latency as well as recall total. The theoretical motivation for this study is described later in the introduction, immediately after a review of the relevant literature.

\section{Evidence Against Interference}

Some of the earliest studies in which the effect of a concurrent task on memory retrieval was examined paired a tracking task with the recall phase of a free recall paradigm. Error in the secondary tracking task was clearly increased by the concurrent performance of the recall task, suggesting that memory retrieval is, in fact, a demanding mental task (Johnston, Greenberg, Fisher, \& Martin, 1970; Martin, 1970; Trumbo \& Milone, 1971). 
Yet Baddeley et al. (1984) noted that these studies involved tasks with heavy response-related demands and argued that the observed interference by tracking might reflect conflict in response execution, rather than anything specific to memory retrieval. Hence, Baddeley et al. paired the recall task with different kinds of concurrent tasks, and these tasks coincided with either the study period or the recall period of a standard free recall task. For the concurrent task in two of these studies, for example, subjects sorted a deck of cards by placing cards into one of four piles at a rate of once per $2 \mathrm{sec}$, with the aid of a metronome. The easy condition required subjects to simply place cards facedown into four piles, whereas the difficult condition required subjects to sort the cards into four piles on the basis of suit. Although the difficulty of the sorting task affected recall total when subjects sorted cards during the study period, recall total was unaffected by the difficulty of the sorting task when it coincided with the recall phase. These findings led the authors to conclude that recall total is mostly unaffected when memory retrieval is paired with a demanding concurrent task.

Baddeley et al.'s (1984) findings were confirmed by four recent free recall studies showing that recall total is scarcely affected when the recall period is paired with a demanding concurrent task. In the first study, Craik et al. (1996) found that recall total was mostly unaffected when a serial-choice-RT task was paired with the recall period (whereas a sharp decline was observed when the concurrent task was paired with the study period). In a second experiment, these same authors reported that the protected status of memory retrieval was not greatly affected by a manipulation of emphasis during the instructions (i.e., memory task, choice-RT task, or 50/50). In the third study, Anderson et al. (1998) replicated the null effect of a serialchoice-RT task on recall total with both younger and older adults. In the final study, Naveh-Benjamin et al. (1998) corroborated the basic findings with a fine-grained analysis of the specific costs to both memory and the concurrent task. These intriguing null effects of a concurrent task on the recall phase led these researchers to describe memory retrieval as "resilient," "protected," and even "obligatory."

\section{Evidence for Interference}

Whereas the free recall studies described above indicate that memory retrieval is protected from an interfering concurrent task, a different conclusion is given by the results of two dual-task studies incorporating a different memory task. The first of these incorporated the psychological refractory period (PRP) design. Here, two punctate tasks are performed on each trial, with a variable stimulus onset asynchrony (SOA) separating the stimuli for the two tasks. A ubiquitous finding (usually termed the PRP effect) is an increase in Task 2 RTs as SOA is shortened (Welford, 1952, 1980; see Pashler, 1998, for a review). Carrier and Pashler (1995) reported a PRP experiment in which the first task involved making a manual response to the pitch of a tone, whereas the second task required a cued (pairedassociate) retrieval with a visually presented cue word and a vocal response. The cued recall task was much more time consuming than the choice-RT task-about $1,350 \mathrm{msec}$ versus less than $600 \mathrm{msec}$. Therefore, if the two tasks could be carried out independently, responses would rarely have occurred in very close temporal proximity. Nonetheless, cued recall RT increased as the SOA was shortened. Furthermore, when the difficulty of the memory retrieval was increased, the effect on cued recall RT combined additively with the effect of SOA. This additivity is as predicted by the hypothesis that memory retrieval is postponed by the concurrent task, and it invalidates the hypothesis that the slowing reflects conflicts in the execution of motor responses (see Pashler, 1994a, 1998; but see Hommel, 1998). The Carrier and Pashler findings suggest that while centrally demanding stages of the first task are underway (e.g., selecting a response to the tone), retrieval of the target word is postponed. Thus, the results suggest not merely that there is interference between memory retrieval and another task, but that this interference is total in character.

A similar conclusion is given by the results of a study requiring subjects to generate exemplars of a semantic category (e.g., kinds of fruits) while concurrently responding to a serial-choice-RT task (Rohrer et al., 1995). In this study, the presence of the concurrent-choice-RT task depressed recall totals by about $20 \%$. Moreover, the mean recall latency of these responses, where latency is measured from the onset of the recall period, was increased by more than $80 \%$. Notably, an unpublished first version of this study revealed virtually no effect of the concurrent task on either recall total or recall latency, and this first study differed from the published version in only one respect. In the serial-choice-RT task used in the first study, an asterisk appeared in one of three positions within a row, and subjects depressed the corresponding key from among three adjacent keys in the same row of the keyboard (i.e., left-left, middle-middle, right-right). This mapping between stimulus and response is trivial, thereby minimizing the difficulty of the response selection stage of the choice-RT task. Because the response selection stage has been shown to be the centrally demanding portion of this task (cf. Pashler, 1994a, 1998), the serialchoice-RT task in the first study may not have placed sufficiently taxing demands on the central processes needed by memory retrieval. For this reason, the choice-RT task in the second study was altered so that the stimulus included one, two, or three asterisks and subjects responded by pressing one of three keys, depending on the total number of presented asterisks. Here, the use of an arbitrary stimulus-response mapping increased the duration of the centrally demanding response selection process of the concurrent task, and this presumably caused the observed impairment in concurrent memory retrieval.

\section{Reconciling the Conflicting Studies}

How should one reconcile the apparently protected status of retrieval, noted in the free recall paradigms, with the postponement of memory retrieval suggested by the stud- 
ies incorporating the PRP design or semantic memory retrieval? A number of accounts might be proposed, and two seem particularly deserving of consideration.

The first possibility is that the different results reflect the choice of memory paradigm. The category exemplar study described above (Rohrer et al., 1995), for instance, relied on semantic memory, whereas free recall is an episodic memory task. Likewise, whereas the free recall paradigm requires continuous retrieval of multiple items, the PRP study utilized discrete retrievals. Hence, the interference observed with the discrete PRP tasks may be a special case not reflective of dual-task performance in general. For example, isolated stimuli may "seize" resources beyond those required to process them, thereby interfering with subsequent performance of other tasks. On this sort of account, results obtained with the PRP design would be misleading, in the sense that that they would understate the potential for parallel processing in situations that are more continuous (Neumann, 1987).

The second possibility is that the free recall studies were misleading because all of these studies relied on a concurrent task that may have placed insufficient demands on the central processes needed for memory retrieval. Specifically, the proportion of time during the recall period devoted to the centrally demanding stages of the concurrent task may have been too small. We term this proportion the temporal density. When the concurrent task has low temporal density, there are many opportunities to carry out processing operations that would likely have little effect on memory retrieval, be it "protected" or not. Hence, the apparent "protected" status of memory retrieval suggested by these free recall studies might reflect the absence of a serious challenge by a competing task, rather than its ability to withstand such a challenge. In fact, the four recall experiments used the identical concurrent task (Experiment 1 in Anderson et al., 1998; Experiments 1 and 2 in Craik et al., 1996; Experiment 2 in Naveh-Benjamin et al., 1998). In this serial-choice-RT task, an asterisk would appear in one of four positions within a row, and these stimuli were assigned to the four horizontally adjacent response keys by vertical alignment (i.e., left-left, left-center-left-center, right-center-right-center, right-right). With practice, this high stimulus-response compatibility ensures an easy response selection process and, thereby, minimizes the centrally demanding proportion of the task (Pashler, 1998). Moreover, previous research has sometimes indicated that highly compatible stimulus-response pairs generate no detectable central interference at all (e.g., Greenwald \& Shulman, 1973; McLeod \& Posner, 1984; Pashler, Carrier, \& Hoffman, 1993; Schumacher et al., 2001). In addition to the highly compatible stimulus-response mapping, the serial-choice-RT task in these previous studies utilized self-pacing. That is, each stimulus was triggered by the subject's response to the previous stimulus, and this self-pacing may have allowed subjects to "cheat" the concurrent task at times when memory retrieval was particular challenging. In fact, performance on the serialchoice-RT task was dramatically slowed by memory retrieval (in comparison with a control condition in which subjects performed only the serial-choice-RT task). In a similar fashion, Hicks and Marsh (2000) found that the impact of a concurrent task on a recognition test depends critically on the demands of the concurrent task. In summary, then, the null effects of a concurrent task on memory retrieval observed in the aforementioned free recall studies might simply reflect the use of an insufficiently distracting concurrent task.

\section{Present Research}

To test these competing explanations, we paired the recall phase of a free recall paradigm with a concurrent task with greater demands on central processes (i.e., a task with greater temporal density). In particular, we utilized a serial-choice-RT task that differed from those used in the previous recall studies in two ways. First, the stimulusresponse mappings were not highly compatible. The stimulus was a red, blue, or green square, and the subjects responded by depressing one of three preassigned keys on the keyboard. Notably, this task is an easy one, especially after the 10 min of practice each subject completed, but the identity of the correct response is not inherently suggested by the location of the stimulus. Second, the serialchoice-RT task was presented with a constant interstimulus interval (ISI), rather than with a constant interresponse interval (IRI), in keeping with an early method used by Kalsbeek and Sykes (1967). Hence, the serial-choice-RT stimuli appeared at a rate that was fixed rather than variable. The duration of the ISI was determined on the basis of the subject's prior performance, and this calibration was such that the subject could almost always respond before the next stimulus was presented but would readily fall behind if gross delays occurred.

In addition to theses changes in the concurrent task, the present study included an analysis of the time course of retrieval, in addition to the usual tabulation of recall total. A measure of latency is critical, of course, because a null effect on recall total does not ensure a null effect on recall latency. In effect, someone may be able to walk while chewing gum, but this feat is not particularly impressive if the gum chewing hinders walking speed.

The time course of free recall is principally assessed by the measure of mean recall latency. The latency of each recalled word equals the time elapsed between the beginning of the recall period and the vocal onset of the response, and mean recall latency simply equals the arithmetic mean of the latencies. For example, if a subject recalls three words with latencies of 5,10 , and $30 \mathrm{sec}$, mean recall latency for that trial equals $15 \sec [(5+10+30) / 3]$. Likewise, mean recall latency can be calculated directly by simply averaging recall latencies across trials and subjects. Alternatively, mean recall latency can also be measured by a parameter estimate given by a curve-fitting technique described in the Results section, and previous researchers have uniformly relied on these parameter estimates when measuring recall latency (e.g., Bousfield, Sedgewick, \& Cohen, 1954; Gronlund \& Shiffrin, 1986; 
Roediger, Stellon, \& Tulving, 1977; Rohrer, 1996; Rohrer \& Wixted, 1994; Wixted \& Rohrer, 1994). The present study includes values of mean recall latency obtained by both direct calculation and curve fitting, and both techniques yielded the same conclusion.

\section{METHOD}

\section{Subjects}

Twelve college undergraduates participated in return for course credit.

\section{Materials}

Each word list included 15 monosyllabic four-letter words, with no word appearing on more than one list. The word order for each list was randomized but did not vary across subjects. Two word lists were used for practice trials, and eight different word lists were used in scored trials. Each of the latter eight word lists appeared equally often in the two conditions utilizing the recall task (i.e., the recallonly condition and the dual-task condition).

\section{Design}

Each subject completed four sets of three trials each (excluding the practice trials described in the Procedure section). Each set began with one trial of the keypress-only condition. The second and third trials in each set represented the recall-only and dual-task conditions. The recall-only condition preceded the dual-task condition during the first and third sets for one half of the subjects and during the second and fourth sets for the remaining subjects.

\section{Procedure}

The subjects were tested one at a time in the presence of an experimenter. The subjects began each session by speaking into the voice key microphone for a 60 -sec period, in order to become familiar with its operation. The subjects then completed two 5-min practice sessions with the keypress task and then completed 1 practice trial in the recall-only condition and 1 practice trial in the dualtask condition. The subjects then completed the 12 scored trials.

The keypress task was a serial-choice-RT task of 60-sec duration, with the subjects responding to each of the continuously appearing stimuli. Each stimulus was a red, green, or blue square that appeared for $200 \mathrm{msec}$, and each square had sides measuring $3.8 \mathrm{~cm}$ and subtending a $3.11^{\circ}$ visual angle at a viewing distance of about $70 \mathrm{~cm}$. The subjects responded by pressing one of three preassigned adjacent keys on the keyboard ("B" for red, " $\mathrm{N}$ " for green, and "M" for blue). The subjects were instructed to rest their first three fingers on these keys throughout the task and to "respond to each color as quickly but as accurately as possible." The ISI varied across subjects. In the first 5-min practice period, the ISI was set to $1,300 \mathrm{msec}$. In the second 5-min practice period, the ISI was set equal to the 90th percentile of that subject's RTs from the last minute of the first practice period. In the scored trials, the ISI equaled the 90th percentile of the subject's RTs from the last minute of the second 5-min practice session.
The recall task included a study period, a distractor task, and a recall period. During study, 15 words were presented serially at a rate of once per $4 \mathrm{sec}$, and the subjects read each word aloud. In the immediately following 34-sec distractor task, the subjects counted backward by threes from a randomly chosen three-digit number that appeared on the screen. Next, two question marks cued the beginning of the 60 -sec recall phase, and the subjects then attempted to recall the most recent list of 15 study words in any order. In the dualtask condition, the 60 -sec recall period coincided with the 60 -sec keypress task, and the first colored square appeared when the recall period began.

A voice key and a computer measured the voice onset of each recalled word. An experimenter monitored these recall latencies in real time and noted the latency corresponding to each incorrect response. The experimenter also recorded the latencies of voice key false alarms reflecting extraneous noises, such as a cough or an errant word. The latencies for both incorrect responses and voice key false alarms were removed from the data before subsequent analyses. In addition, each session was recorded on audiotape.

\section{RESULTS}

\section{Keypress Task}

Performance on the keypress task was significantly impaired by the concurrent recall task, as evidenced by a comparison of the keypress-only condition and the dualtask condition. Specifically, concurrent recall reduced mean accuracy in the keypress task from .91 to $.81[t(11)=3.70$, $p<.01]$, while increasing the mean RT of the correct responses from 476 to $512 \mathrm{msec}[t(11)=3.36, p<.01]$. These means and the associated standard errors are listed in Table 1.

\section{Recall Task}

Recall was sharply impaired by the concurrent keypress task, since both recall total and recall latency were worse in the dual-task condition than in the recall-only condition. Specifically, the concurrent keypress task significantly reduced mean recall total per trial from 7.56 to 5.67 words $[t(11)=3.57, p<.01]$ and increased the mean recall latency of these correct responses from 12.58 to $17.49 \mathrm{sec}$ $[t(11)=4.28, p<.01]$. These means and their standard errors are listed in Table 1. In short, the concurrent task reduced recall total by $25 \%$ and increased recall latency by about $40 \%$.

This impairment of recall is well illustrated by the cumulative recall data shown in Figure 1A. These data represent the accumulation of recall total as a function of time elapsed during the recall period, with each data point corresponding to a 1 -sec interval. These curves grow ever

Table 1

Results

\begin{tabular}{|c|c|c|c|c|c|c|c|c|}
\hline \multirow[b]{3}{*}{ Condition } & \multicolumn{4}{|c|}{ Recall } & \multicolumn{4}{|c|}{ Keypress } \\
\hline & \multicolumn{2}{|c|}{ Total } & \multicolumn{2}{|c|}{ Latency $(\mathrm{sec})$} & \multicolumn{2}{|c|}{ Accuracy } & \multicolumn{2}{|c|}{ RT (msec) } \\
\hline & $M$ & $S E$ & $M$ & $S E$ & $M$ & $S E$ & $M$ & $S E$ \\
\hline Recall only & 7.56 & 0.68 & 12.58 & 0.79 & - & - & - & - \\
\hline Button only & - & - & - & - & .909 & .008 & 476 & 18 \\
\hline Dual task & 5.67 & 0.56 & 17.49 & 1.52 & .813 & .027 & 512 & 13 \\
\hline
\end{tabular}

Note-All four pairwise comparisons are statistically significant $(p<.01)$. 

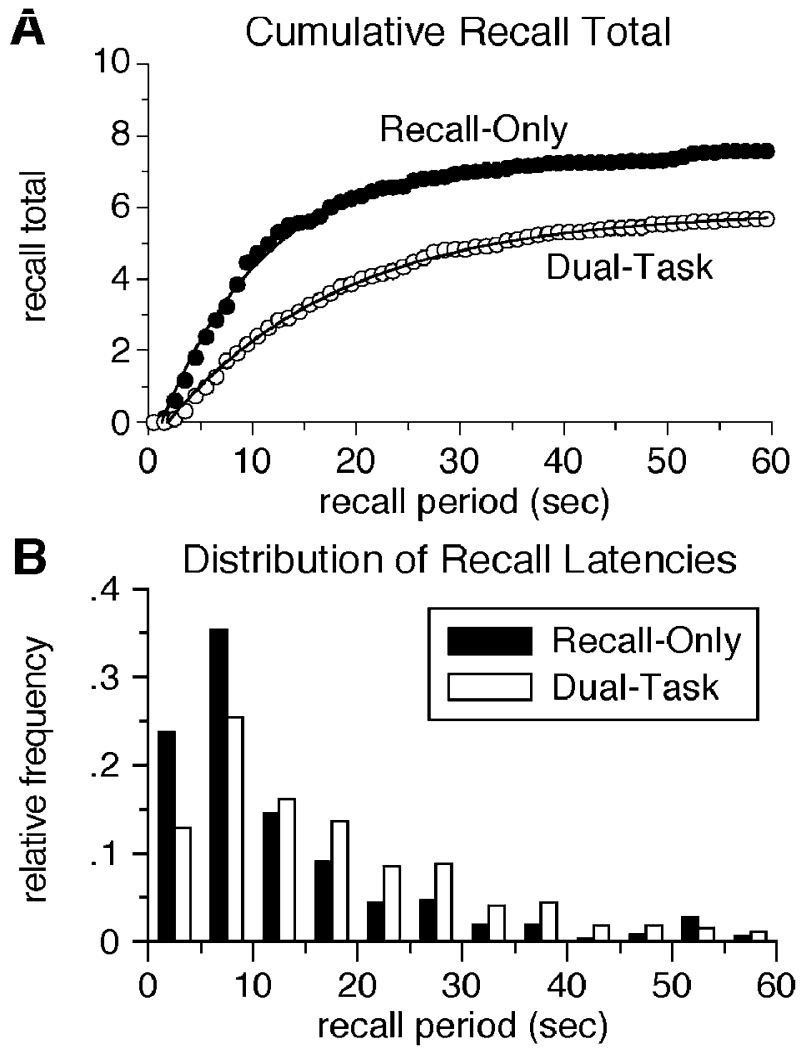

Figure 1. Recall data. (A) Cumulative recall curves. These data represent mean recall total (per trial) as a function of time elapsed during the recall period, and the solid lines indicate the best-fitting cumulative exponential (Equation 1). The ultimate heights of these curves reveal that the concurrent task reduced recall total from about 7.5 to 5.5 , and the more gradual shape of the dual-task curve reveals that the concurrent task increased mean recall latency. For example, the recall-only curve reaches one half of its ultimate height in less than $10 \mathrm{sec}$, whereas the dual-task curve does not reach its shorter halfway height until after 10 sec. (B) Recall latency distributions. The concurrent task effect on recall latency is best illustrated by comparing the distribution of latencies. Here, the height of each vertical bar reflects the proportion of responses given in the corresponding 5 -sec interval. The first 5-sec interval, for example, included about $24 \%$ of the recalled items in the recall-only condition but only about $13 \%$ of the recalled items in the dual-task condition. Beyond the 10 -sec mark, however, the dual-task proportions exceed the recall-only proportions. Hence, the recall latencies were right-shifted by the concurrent keypress task, despite the fact that the subjects recalled fewer words in this dual-task condition.

more gradual as subjects recall words at an increasingly slower rate before eventually reaching the ultimate recall total. Hence, the recall-only curve climbs to an ultimate height of about 7.5, whereas the dual-task curve eventually reaches a height of about 5.5. These curves also reveal differences in recall latency. In particular, the initial slope of the recall-only curve is much greater than that of the dual-task curve, and this is true even when this growth rate is measured in proportion to the ultimate recall total. For instance, the recall-only curve climbs to a height of 3.75about half of its ultimate recall total-before the 10 -sec mark. Yet the dual-task curve does not reach its shorter halfway height of about 2.75 until well after the 10 -sec mark.

As was noted in the introduction, these cumulative recall curves provide a parameter estimate of mean recall latency when a cumulative exponential is fit to these data. The cumulative exponential is given mathematically by the expression

$$
F(t)=a\left(1-e^{-(1 / \tau)(\mathrm{t}-\mathrm{c})}\right),
$$

where $a$ represents the asymptotic recall total, $c$ represents a horizontal time shift to account for any delay at the beginning of the recall period, and $\tau$ represents mean recall latency. (Technically, many researchers use an alternate form of this equation that replaces $\tau$ with the reciprocal of the so-called growth parameter, but this difference is merely superficial.)

Figure 1A includes the least-square fits of the cumulative exponentialfor both sets of cumulative recall data. These curves do not begin to rise until almost $2 \mathrm{sec}$ after the recall period begins, and this shift reflects the typical pause preceding the first response in free recall. Consequently, the parameter estimates of mean recall latency exclude the duration of the pause (unlike the direct calculation method). These estimates equaled $10.08 \mathrm{sec}$ in the recall-only condition and $16.82 \mathrm{sec}$ in the dual-task condition. Hence, the concurrent task increased the parameter estimate of mean recall latency by $67 \%$ (as compared with the $40 \%$ increase revealed by the directly calculated latencies described above and listed in Table 1).

Finally, the effect of the keypress task on mean recall latency is also well illustrated by the relative frequency distributions shown in Figure 1B. In this plot, the height of each bar indicates the proportion of responses given in the corresponding 5-sec interval. Hence, the first 5-sec interval included about $24 \%$ of the recalled words in the recallonly condition and about $13 \%$ of the recall words in the dual-task condition. A visual comparison of the two distributions reveals that the temporal distribution of recall latencies was shifted sharply to the right by the concurrent keypress task. In summary, each of the recall latency analyses reveals that recall was dramatically slowed by the keypress task.

\section{DISCUSSION}

In the introduction, it was pointed out that the most careful studies of the effect of performing a dissimilar concurrent task on continuous free recall tasks have revealed scarcely any impairment in recall total. By contrast, when the effect of this concurrent task had been measured with a semantic retrieval task or a discrete dual-task (PRP) design, the results suggested total (or virtually total) interference between retrieval and the centrally demanding cognitive operations within the concurrent task.

The results of the present study generally support the latter findings, indicating that memory retrieval is subject to severe interference from unrelated central processing. In particular, performing a series of unrelated choice-RT 
tasks was shown to reduce recall total and drastically impede the speed of recall in a free recall task, even though the concurrent task did not involve verbal material. The discrepancy between the present result and the findings of previous free recall studies appears to reflect the temporal density of the concurrent task, since the present study utilized a serial-choice-RT task designed to ensure that the subjects devoted a substantial proportion of their time to centrally demanding phases of the concurrent task. Specifically, stimulus-response compatibility was less than maximal (being based on color-position rather than positionposition-linkages), thus increasing the difficulty and duration of the centrally demanding stage of response selection. In addition, the serial-choice-RT task in the present study relied on a constant ISI, rather than on a constant IRI; this constant pacing of the stimuli prevents subjects from cheating the concurrent task whenever memory retrieval is particularly challenging.

Another important feature of the present data is that the concurrent task impaired not only retrieval speed, but recall total as well (by about 25\%). It appears, then, that the concurrent task did not merely slow the retrieval of the ultimately recalled items but actually precluded some items from being retrieved at all. This result raises several interesting possibilities. First, the momentary demands of retrieval may vary dramatically across items (cf. Naveh-Benjamin $\&$ Guez, 2000). Second, the retrieval of some items may require relatively long periods of uninterrupted effort, and these longer uninterrupted durations may simply be unavailable when the concurrent task competes with memory retrieval at regular intervals. Third, the retrieval of some items may require a kind of momentum that accumulates during an immediately preceding quick succession of one or two successful retrievals, and such momentum may be prevented by a concurrent task.

Furthermore, it seems unlikely that this dual-task interference can be explained by response-related interference between the concurrent task and the overt production of responses. First, the concurrent task in the present study involved a manual response, whereas the retrieval task required spoken responses. As even casual observation reveals, people have little difficulty speaking while making manual responses (McLeod \& Mierop, 1979; Van Galen \& ten Hoopen, 1976). Second, even if there did exist serious interference between these motoric responses, the subjects devoted very little time to responding in the recall task. Specifically, the subjects in the dual-task condition recalled an average of just six words, so the time spent producing this small number of four-letter monosyllabic words would have occupied no more than 2 or $3 \mathrm{sec}$ of the 60 -sec recall period.

The role of temporal density is further evidenced by the results of a second study that utilized the choice-RT task used in the previous free recall studies showing little effect of the concurrent task on recall total (Experiment 1 in Anderson et al., 1998; Experiments 1 and 2 in Craik et al., 1996; Experiment 2 in Naveh-Benjaminet al., 1998). Specifically, the stimulus appeared in one of three locations within a row, and these stimuli were assigned to the corresponding three adjacent keys within one row of the keyboard. In addition, the stimuli appeared at a constant rate. Hence, a highly compatible stimulus-response pairing ensured a trivial response selection, and the experimenterpaced stimuli precluded brief respites from the distractor task. As was described in the introduction, both features reduce the proportion of time devoted to the centrally demanding processes of the concurrent task. In keeping with the results of the previous studies, the concurrent task reduced recall total by only $2 \%[7.40$ vs. $7.22 ; t(11)=0.42$, n.s.]. Likewise, recall latency, which had not been reported in the previous studies, increased by only $8 \%$ $[11.08$ vs. $12.00 ; t(11)=1.17$, n.s.]. Because both measures yielded null effects, the study is presented informally here. Nevertheless, a comparison of this finding with the main experiment reported above provides further evidence for the view that the interfering effect of the concurrent task depends critically on its temporal density.

What remains unclear, however, is whether the interference suffered by memory retrieval is as severe as the PRP results have been taken to suggest, with memory retrieval completely blocked at those times when central processing on the other task is underway (supporting a time-sharing, rather than a capacity-sharing, analysis). Superficially, the present results might seem to reject a time-sharing account, because the rate of recall in the present study was not reduced to zero. However, even though stimuli in the concurrent task were presented at a rapid continuous rate with experimenter pacing, the centrally demanding stages of the concurrent task were virtually certain to occupy no more than a sizable fraction of the total time consumed by the task. Thus, there would undoubtedly have been many brief periods of slack while the motoric or perceptual, rather than the central, stages of the concurrent task were underway. In fact, results from PRP studies would suggest that competing tasks can proceed during these times. (The reality of these slack periods is clearly revealed in the advantage conferred by stimulus preview in continuouschoice-RT tasks; cf. Pashler, 1994b.) Given that such slack did exist in the present study, the outcome is consistent with the possibility that retrieval was indeed completely (albeit intermittently) blocked by the concurrent task. At the very least, however, the results suggest that retrieval was substantially slowed by the concurrent task, an outcome not in keeping with a description of retrieval as automatic, protected, or obligatory.

The demands of memory retrieval can also be elucidated by dual-task studies requiring two memory retrieval tasks, and these studies generally find evidence of gross interference when subjects attempt to concurrently retrieve two items that belong to different categories. Such interference has been observed when subjects recall words from a list spanning two categories (Rohrer, Pashler, \& Etchegaray, 1998) and when subjects generate exemplars from presented categories (Maylor, Chater, \& Jones, 2001). Likewise, two overlapping lexical decision trials yield interference when the two words belong to different cate- 
gories (Logan \& Schulkind, 2000). It appears that the notion of a category can be defined broadly, since Logan and Delheimer (2001) reported data revealing parallel retrieval in a yes-no recognition task when the two study words shared spatial or temporal proximity in the study phase. In summary, these studies suggest that the cost of memory retrieval reflects the number of categories under search, whether these categories are defined by semantic relatedness or episodic proximity.

Finally, although the present results clearly challenge the idea that memory retrieval is obligatory or protected in any strong sense, the previous free recall studies nevertheless have indicated that memory retrieval is typically impaired to a lesser degree than performance on the concurrent task. Nothing we have stated so far would explain this intriguing asymmetry. One way to reconcile the asymmetry with the present findings might be to suppose that although retrieval is neither obligatory nor protected, it is difficult for executive processes to interrupt memory retrieval in the absence of new stimulation. That is, these interruptions might be costly, a possibility suggested by sophisticated analyses of interruption effects presented by Byrne (2000). One possible explanation for this cost might be that self-interruption requires reliance on temporal information ("setting a mental stopwatch"), and memory retrieval itself may utilize some of the mechanisms required for that form of timekeeping. This is, of course, mere conjecture, but it would appear to be potentially testable.

\section{REFERENCES}

Anderson, N. D., Craik, F. I. M., \& Naveh-Benjamin, M. (1998). The attentional demands of encoding and retrieval in younger and older adults: I. Evidence from divided attention costs. Psychology \& Aging, 13, 405-423.

Baddeley, A., Lewis, V., Eldridge, M., \& Thomson, N. (1984). Attention and retrieval from long-term memory. Journal of Experimental Psychology: General, 113, 518-540.

Bousfield, W. A., Sedgewick, C. H., \& Cohen, B. W. (1954). Certain temporal characteristics of the recall of verbal associates. American Journal of Psychology, 67, 111-118.

Byrne, M. D. (2000). Are retrievals from long-term memory interruptible? In L. R. Gleitman \& A. K. Joshi (Eds.), Proceedings of the TwentySecond Annual Conference of the Cognitive Science Society (pp. 7176). Mahwah, NJ: Erlbaum.

Carrier, L. M., \& Pashler, H. (1995). Attentional limits in memory retrieval. Journal of Experimental Psychology: Learning, Memory, \& Cognition, 21, 1339-1348.

Craik, F. I. M., Govoni, R., Naveh-Benjamin, M., \& Anderson, N. D. (1996). The effects of divided attention on encoding and retrieval processes in human memory. Journal of Experimental Psychology: General, 125, 159-180.

Craik, F. I. M., Naveh-Benjamin, M., Ishaik, G., \& Anderson, N. D. (2000). Divided attention during encoding and retrieval: Differential control effects? Journal of Experimental Psychology: Learning, Memory, \& Cognition, 26, 1744-1749.

Fernandes, M. A., \& Moscovitch, M. (2000). Divided attention and memory: Evidence of substantial interference effects at retrieval and encoding. Journal of Experimental Psychology: General, 129, 155-176.

Greenwald, A. G., \& Shulman, H. G. (1973). On doing two things at once: II. Elimination of the psychological refractory period effect. Journal of Experimental Psychology, 101, 70-76.

Gronlund, S. D., \& Shiffrin, R. M. (1986). Retrieval strategies in recall of natural categories and categorized lists. Journal of Experimental Psychology: Learning, Memory, \& Cognition, 12, 550-561.

Hicks, J. L., \& MARSH, R. L. (2000). Toward specifying the attentional demands of recognition memory. Journal of Experimental Psychology: Learning, Memory, \& Cognition, 26, 1483-1498.

Hommel, B. (1998). Automatic stimulus-response translation in dualtask performance. Journal of Experimental Psychology: Human Perception \& Performance, 24, 1368-1384.

iddaka, T., Anderson, N. D., Kapur, S., Cabeza, R., \& Craik, F. I. M. (2000). The effect of divided attention on encoding and retrieval in episodic memory revealed by positron emission tomography. Journal of Cognitive Neuroscience, 12, 267-280.

Johnston, W. A., Greenberg, S. N., Fisher, R. P., \& Martin, D. W. (1970). Divided attention: A vehicle for monitoring memory processes. Journal of Experimental Psychology, 83, 164-171.

Kalsbeek, J. W., \& Sykes, R. N. (1967). Objective measurement of mental load. Acta Psychologica, 27, 253-261.

Logan, G. D., \& Delheimer, J. A. (2001). Parallel memory retrieval in dual-task situations: II. Episodic memory. Journal of Experimental Psychology: Learning, Memory, \& Cognition, 27, 668-685.

Logan, G. D., \& Schulkind, M. D. (2000). Parallel memory retrieval in dual-task situations: I. Semantic memory. Journal of Experimental Psychology: Human Perception \& Performance, 26, 1072-1090.

Martin, D. W. (1970). Residual processing capacity during verbal organization in memory. Journal of Verbal Learning \& Verbal Behavior, 9, 391-397.

Maylor, E. A., Chater, N., \& Jones, G. V. (2001). Searching for two things at once: Evidence of exclusivity in semantic and autobiographical memory retrieval. Memory \& Cognition, 29, 1185-1195.

McLeod, P., \& Mierop, J. (1979). How to reduce manual response interference in the multiple task environment. Ergonomics, 22, 469-475.

McLeod, P., \& Posner, M. I. (1984). Privileged loops from percept to act. In H. Bouma \& D. G. Bouwhuis (Eds.), Attention and performance $X$ : Control of language processes (pp. 55-66). London: Erlbaum.

Naveh-Benjamin, M., Craik, F. I. M., Gavrilescu, D., \& Anderson, N. D. (2000). Asymmetry between encoding and retrieval processes: Evidence from divided attention and a calibration analysis. Memory \& Cognition, 28, 965-976.

Naveh-Benjamin, M., Craik, F. I. M., Guez, J., \& Dori, H. (1998). Effects of divided attention on encoding and retrieval processes in human memory: Further support for an asymmetry. Journal of Experimental Psychology: Learning, Memory, \& Cognition, 24, 1091-1104.

Naveh-Benjamin, M., Craik, F. I. M., Perretta, J. G., \& Tonev, S. T. (2000). The effects of divided attention on encoding and retrieval processes: The resiliency of retrieval processes. Quarterly Journal of Experimental Psychology, 53A, 609-625.

Naveh-Benjamin, M., \& Guez, J. (2000). Effects of divided attention on encoding and retrieval processes: Assessment of attentional costs and a componential analysis. Journal of Experimental Psychology: Learning, Memory, \& Cognition, 26, 1461-1482.

Neumann, O. (1987). Beyond capacity: A functional view of attention. In H. Heuer \& A. Sanders (Eds.), Perspectives on perception and action (pp. 361-394). Hillsdale, NJ: Erlbaum.

Park, D. C., Smith, A. D., Dudley, W. N., \& Lafronza, V. N. (1989). Effects of age and a divided attention task presented during encoding and retrieval on memory. Journal of Experimental Psychology: Learning, Memory, \& Cognition, 15, 1185-1191.

PAshler, H. (1994a). Dual-task interference in simple tasks: Data and theory. Psychological Bulletin, 116, 220-244.

PASHLER, H. (1994b). Overlapping mental operations in serial performance with preview. Quarterly Journal of Experimental Psychology, 47A, 161-191.

PASHLER, H. (1998). The psychology of attention. Cambridge, MA: MIT Press.

Pashler, H., Carrier, M., \& Hoffman, J. (1993). Saccadic eye movements and dual-task interference. Quarterly Journal of Experimental Psychology, 46A, 51-82.

Roediger, H. L., III, Stellon, C. C., \& Tulving, E (1977). Inhibition from part-list cues and rate of recall. Journal of Experimental Psychology: Human Learning \& Memory, 3, 164-188.

Rohrer, D. (1996). On the relative and absolute strength of a memory trace. Memory \& Cognition, 24, 188-201.

Rohrer, D., Pashler, H., \& Etchegaray, J. (1998). When two memories can and cannot be retrieved concurrently. Memory \& Cognition, 26, 731-739. 
Rohrer, D., \& WiXted, J. T. (1994). An analysis of latency and interresponse time in free recall. Memory \& Cognition, 22, 511-524.

Rohrer, D., Wixted, J. T., Salmon, D. P., \& Butters, N. (1995). Retrieval from semantic memory and its implications for Alzheimer's disease. Journal of Experimental Psychology: Learning, Memory, \& Cognition, 21, 1127-1139.

Schumacher, E. H., Seymour, T. L., Glass, J. M., Fencsik, D. E., Lauber, E. J., Kieras, D. E., \& Meyer, D. E. (2001). Virtually perfect time sharing in dual-task performance: Uncorking the central cognitive bottleneck. Psychological Science, 12, 101-108.

Troyer, A. K., Winocur, G., Craik, F. I. M., \& Moscovitch, M. (1999). Source memory and divided attention: Reciprocal costs to primary and secondary tasks. Neuropsychology, 13, 467-474.
Trumbo, D., \& Milone, F. (1971). Primary task performance as a function of encoding, retention, and recall in a secondary task. Journal of Experimental Psychology, 91, 273-279.

Van Galen, G. P., \& Ten Hoopen, G. (1976). Speech control and single channelness. Acta Psychologica, 40, 245-255.

Welford, A. T. (1952). The "psychological refractory period" and the timing of high speed performance: A review and a theory. British Journal of Psychology, 43, 2-19.

Welford, A. T. (1980). The single-channel hypothesis. In A. T. Welford (Ed.), Reaction time (pp. 215-252). New York: Academic Press.

WiXted, J. T., \& Rohrer, D. (1994). Analyzing the dynamics of free recall: An integrative review of the empirical literature. Psychonomic Bulletin \& Review, 1, 89-106.

(Manuscript received December 16, 2000;

revision accepted for publication February 15, 2002.) 